NASA-TM- 85956

NASA Technical Memorandum 85956

NASA-TM-85956 19840018454
Airborne Astronomy Program Medium Altitude Missions Branch Preprint Series 015

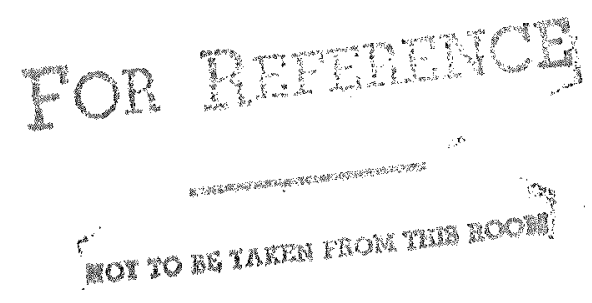

\title{
$183 \mathrm{GHz}$ Water Line Variation: An Energetic Outburst in Orion KL
}

T.B.H. Kuiper, E.N. Rodriguez Kuiper, P.N. Swanson, Dale F. Dickinson, M.J. Klein, and P. Zimmermann

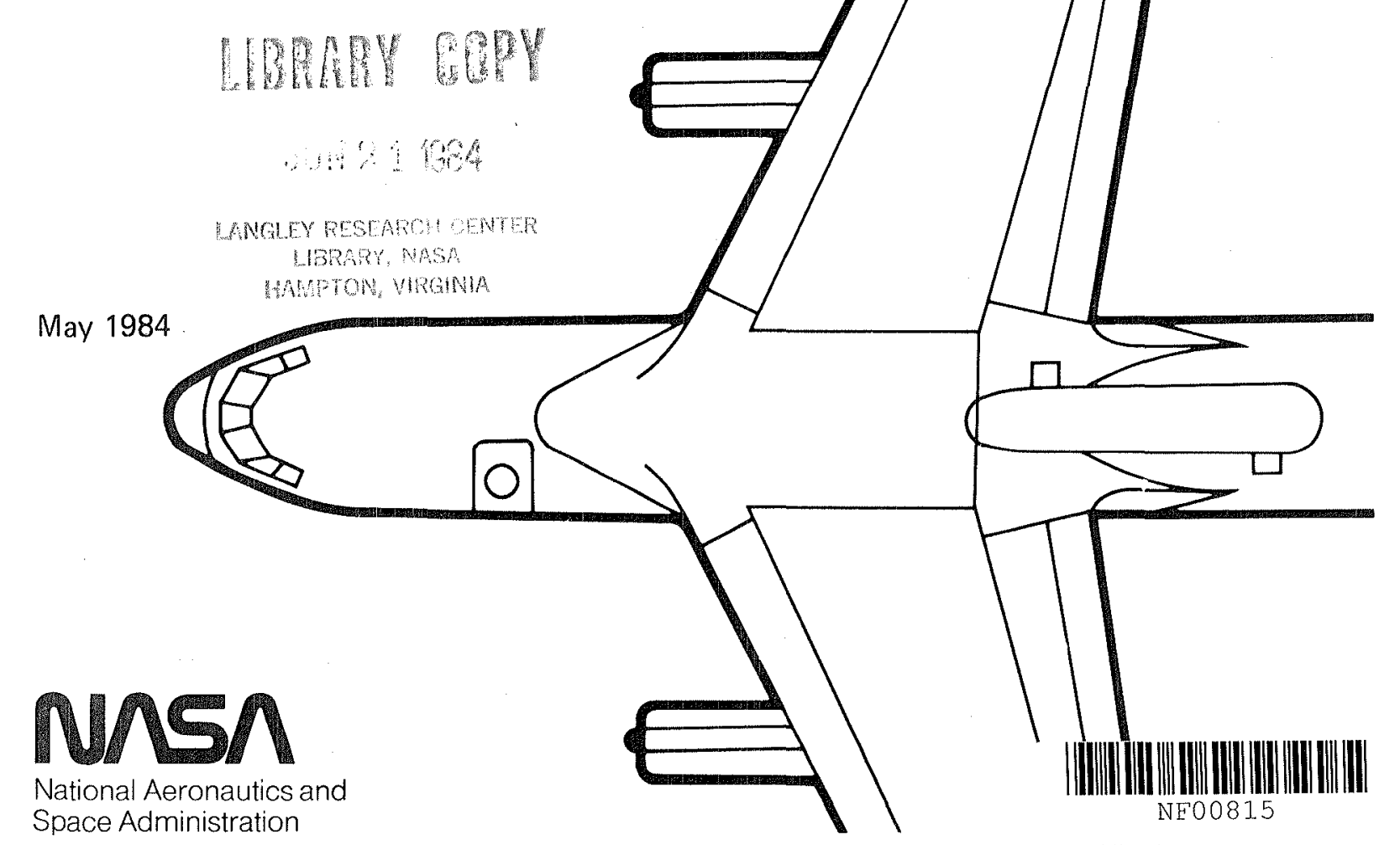




\section{$183 \mathrm{GHz}$ Water Line Variation: An Energetic Outburst in Orion KL}

T. B. H. Kuiper

E. N. Rodriguez Kuiper

P. N. Swanson

Dale F. Dickinson

M. J. Klein

P. Zimmermann, California Institute of Technology

\section{N/SA}

National Aeronautics and

Space Administration

Ames Research Center

Moffett Field, California 94035 
$183 \mathrm{GHz}$ WATER LINE VARIATION:

AN ENERGETIC OUTBURST IN ORION KL

\author{
T. B. H. Kufper \\ E. N. Rodriguez Kuiper ${ }^{\perp}$ \\ P. N. Swanson \\ Dale F. Dickinson ${ }^{2}$ \\ M. J. Klein \\ P. Zimmermann \\ Jet Propulsion Laboratory \\ Calffornia Institute of Technology
}

Recelved 1983 September 12; accepted 1984 February 7

to appear in

The Astrophysical Journal

Part I, August 1, 1984

1 While an employee of Ball Aerospace System Division working at JPL

2 While a NAS-NRC Senfor Resident Research Associate at JPL 


\section{ABSTRACT}

Observations of the $313-220$ transition of water vapour in the direction of Ori $\mathrm{MCl}$ in 1980 February show a $50 \%$ flux increase and an apparent additional red shift of approximately $2 \mathrm{~km} \mathrm{~s}^{-1}$ relative to the line observed in 1977 December. We have adapted the mm-wave telescope calibration formalism to suit optical telescopes, and included an explicit relation to aperture efficiency to facilitate the use of extended source calibration data with spatially unresolved sources. From a detailed examination of the amplitude and frequency calibration, it appears unlikely that the effect is due to systematic error. The increase is attributed to the appearance of a new component at a velocity of $12 \mathrm{~km} \mathrm{~s}^{-1}$ with respect to the local standard of rest. The new component also has broad wings. Increased emission from a region in the high-velocity core of Ori MCl can be due either to additional far-IR radiation to pump the $183 \mathrm{GHz}$ transition or to a change in the physical conditions in the gas. We have carried out statistical equilibrium calculations using the large-velocity-gradient formalism to develop a model for the emission and to identify the physical conditions to which the excitation of the $313-202$ transition is sensitive. The calculations support a model in which the gas in the region of enhanced emission is hotter than the dust. The temporal colncidence between the $183 \mathrm{GHz}$ increase and the $22 \mathrm{GHz}$ water maser outburst suggests a common, impulsive cause, which has heated the gas in a part of the HV source, enhancing the emission in both transitions.

Subject headings: interstellar: molecules--masers--stars:

$$
\text { formation-nebulae: Orion - radio sources: lines }
$$




\section{INTRODUCTION}

Among the features which make the core of $\mathrm{Ori} \mathrm{MCl}$ a unique source of molecular line radiation are strong emissions at $183 \mathrm{GHz}$ due to the ${ }^{3} 13$ 220 transition of water vapour (Waters et a1. 1980a) and at $380 \mathrm{GHz}$ due to its ${ }^{4} 14-3_{21}$ transition (Phillips, Kwan, and Huggins 1980). Both lines have a shape simflar to many molecular lines, consisting of a narrow central "spike" surrounded by broad Doppler wings which are referred to as a "plateau" (Zuckerman and Palmer 1975). The wings of the $183 \mathrm{GHz}$ line were much stronger than had been observed unt 11 that time in other molecules. Assuming that the water vapour wings came from a region at least as small as that which gives rise to the wings in $\mathrm{CO}$, it was found that a mild population inversion was required in the $313-202$ transition to explain the observed intensity (Kuiper, Zuckerman, and kodriguez Kuiper 1981). In 1980 February, brief observations at $183 \mathrm{GHz}$ were made of Ori $\mathrm{MCl}$ as an instrumental check while conducting a search for $183 \mathrm{GHz}$ emfssion from late type stars (Kuiper et al. 1984). Relative to the previous observations of 1976 and 1977, the peak antenna temperature was nearly twice as strong. We report these results and consider the extent to which the previous interpretations need to be modified to account for this change.

Water maser emission at $22 \mathrm{GHz}$ is a recognized signpost of star formation. Yet, an understanding of the conditions which give rise to this emission continues to be elusive (e.g., Elitzur 1982). We consider the implications of our observation for the $22 \mathrm{GHz}$ maser outburst which was observed during the same period. 


\section{OBSERVATIONS}

The observations were made with Schottky diode mixer receivers mounted at the bent-Cassegrain focus of the $91 \mathrm{~cm}$ telescope of the G.P. Kuiper Airborne Observatory. The instrumentation for the 1977 observations has been described by Waters et al. (1980a, b). A cooled receiver was used for the observations of Orion in 1980 February and is described in subsection a below.

During the 1977 observations, the calibration measurement consisted of an observation of the moon. The moon was not available in the 1980 February flight series, but was observed during a subsequent flight series in 1980 August (when Orion could not be observed). However, since the telescope did not change at all between these flight series, and the receiver only minimally (see below), we feel that the 1980 August calibrations can be applied to the 1980 February data.

In addition, in 1980 August, we made measurements inside the telescope structure and of the cold sky which, when combined with calculations based on our measured horn power pattern, identified $85 \%$ of the losses in an absolute sense. Since the telescope structure did not change from 1976 to 1980, we feel that the 1977 and 1980 observations of orion can be compared to an uncertainty of $\sim 10 \%$. The lunar observations confirm this for those efficiency factors which are not related to the beamidth. The remaining factor for unresolved sources depends on aperture fllumination which cannot have departed from the designed pattern sufficiently to account for an $80 \%$ change in antenna temperature.

Readers who do not wish to study all the detalls are Invited to skip to subsection $c$, Results. 


\section{a) Instrumentation}

In 1980 February, calibration was accomplished by intercepting the beam to the telescope with a flat plate and diverting it downward to a rotating semi-circular mirror which directed it alternately to an ambient absorber and an absorber immersed in liquid nitrogen. To verify the operation of the spectrometers by measuring the atmospheric $183 \mathrm{GHz}$ emission, we used a different configuration, which we describe below, to make our calibration measurements. However, except for the difference in mounting the receiver to the telescope, the systems were identical.

A simplified diagram of the receiver, the KAO telescope, and optical path (not to scale) are also shown in Figure 1. The detector was a Schottky diode, mounted in a reduced height waveguide and cooled to approximately $15 \mathrm{~K}$ by a closed-cycle hellum refrigerator. Power was coupled from the waveguide mode to the Gaussian beam mode by a small corrugated circular horn and a Teflon lens. The local oscillator power was injected through a quasi-optical, folded Fabry-Perot diplexer. The intermediate frequency was amplified by a gallium arsenide field-effect transistor amplifier, cooled to approximately $80 \mathrm{~K}$. The fixed oscillators used in the frequency conversions were counted at JPL before or after each flight series and found not to differ from their nominal frequencies by more than $2 \mathrm{kHz}$. The data system consists of a 36-channel filter bank with resolution at the center frequency of $1 \mathrm{MHz}$ tapering to $8 \mathrm{MHz}$ at the edges and a 250-channel digital Fourier transform spectrometer with $40 \mathrm{KHz}$ resolution over a $10 \mathrm{MHz}$ bandwidth.

The antenna temperature scale was calibrated relative to the ambient and liquid-nitrogen cooled targets in the receiver. The temperatures of the load were measured by means of attached thermistors. The receiver's 
internal calibration was checked in 1980 August by placing ambient and 1iquid-nitrogen soaked Eccosorb foam targets at the receiver aperture. We concluded that the receiver's internal calibration was accurate to $1 \%$ In slope, and $0.3 \%$ in zero offset, relative to the thermistor temperature readings. The double sideband system noise temperature was typically $900 \mathrm{~K}$ for the operational system.

The internal optical components of the receiver were aligned by shining a laser down the optical axis, replacing the opaque Teflon lenses by small irises. The receiver was aligned with the KAO optics by focusing the image of Polaris on the center of the pressure window. Because of the long focal length and long wavelength, the Schottky diode detector is required to be only within $2 \mathrm{~mm}$ of the optic axis and several $\mathrm{cm}$ from the focus along the optic axis. We believe that the pointing was accurate to $\sim 10$ arcsec. Pointing was verified during both the 1977 and 1980 flights by making a five-point map of Orion, which showed that our. pointing error was less than $1 / 2$ arcmin.

To remove sky and telescope background, beam switching between source and adjacent sky was used, subtracting sky from source in real time. The relative positions of the two beams were reversed in alternate integrations which were then subtracted to remove baseline effects due to asymmetry between the two beams. Any residual baseline effects were due to asymmetry in the system and variations on the timescale of several minutes.

\section{b) Telescope Calibration}

We consider how to apply the overall effictencies determined from observations of the moon in 1976 October and 1980 August to observations of unresolved sources. We consider both the losses in the telescope and 
through the atmosphere.

Following the notation of Kutner and Ulich (1981; hereafter referred to as $\mathrm{KU})$, the change in antenna temperature due to the presence of a source may, for single sideband observations, be written as

$$
\Delta \mathrm{T}_{\mathrm{A}}=n_{1} n_{\mathrm{fss}} n_{\mathrm{c}} \mathrm{T}_{\mathrm{R}} \mathrm{e}^{-\tau_{\mathrm{a}}(\nu) \mathrm{A}}
$$

The $\eta$ 's are efficiency terms, where $0<\eta<1 ; \eta_{1}$ accounts for losses in the telescope that terminate in the ambient temperature surroundings; $\eta_{f s s}$ is the efficiency due to spillover past the secondary (we assume that there is no diffuse scatter from optical reflecting surfaces at our wavelength); $\eta_{c}$ Is the efficlency with which the diffraction beam couples to the source; $\tau_{\mathrm{a}}(\nu)$ is the zenith atmospheric opacity at frequency $\nu$; $A$ is the airmass; and $\mathrm{T}_{\mathrm{R}}$ is the radiation temperature of the source. The telescope efficiency, $\eta_{1}$, can for convenience be divided into three terms

$$
n_{1}=n_{r} n_{\text {int }} n_{\text {mo }}
$$

where $\eta_{r}$ is the efficiency due to ohmic losses in the telescope's reflecting surfaces; $\eta_{\text {int }}$ accounts for losses between the recefver and a point adjacent to the tertiary flat blocking the secondary; $n_{m o}$ is the efficiency of the main optics accounting for blockage of the primary by the secondary and the spider. We ignored blockage by the spider. (If the integral over the "forward hemisphere" in $\mathrm{KU}$ is understood to include only the part of the power pattern which sees the sky, then $n_{i n t} n_{\text {mo }}$ is $n_{\text {rss }}$ in the notation of $\mathrm{KU}$.)

When the source is small relative to the telescope beam, Equation 1 may be rewritten (see Eq. 3 of $\mathrm{KU}$ ) as

$$
\begin{gathered}
\Delta \mathrm{T}_{\mathrm{A}^{\prime}}=\Delta \mathrm{T}_{\mathrm{A}} \mathrm{e}^{\tau_{\mathrm{a}}(\nu) \mathrm{A}}=\frac{\eta_{\mathrm{r}}}{\iint_{4 \pi} \mathrm{P}_{\mathrm{n}}(\Omega) \mathrm{d} \Omega} \mathrm{T}_{\mathrm{R}} \iint_{\Omega \mathrm{s}} \mathrm{B}_{\mathrm{n}}(\Psi) \mathrm{d} \Psi \\
-7-
\end{gathered}
$$




$$
=\frac{n_{r}}{\iint_{4 \pi} P_{n}(\Omega) d \Omega} \frac{\lambda^{2} S}{2 k}=n_{A} A S / 2 k
$$

where $S$ is the flux density and the aperture efficiency, $n_{A}$, has its usual definition:

$$
n_{A}=n_{r} \frac{\lambda^{2} / A}{\iint_{4 \pi} P_{n}(\Omega) d \Omega}=n_{r} \lambda^{2 / A} \Omega_{B}
$$

Because $n_{A}$ changed between observations, we compare a modified antenna temperature

$$
\begin{aligned}
T_{A} / \eta_{A} & =\frac{A}{\lambda^{2}} e^{-\tau_{a} A} T_{R} \iint_{\Omega S} B_{n}(\Psi) d \Psi \\
& =A S e^{-\tau a A} / 2 k
\end{aligned}
$$

(We note in passing that $n_{A}=\eta_{S} \lambda^{2} / A \Omega_{B}$, where $n_{s}=n_{f s s} n_{1}$, the extended source efficiency of $\mathrm{KU}$, is referred to in early literature as the beam efficiency.)

During the flight sertes in 1980 August, we placed ambient temperature and liquid nitrogen-cooled absorbers next to the tertiary so as to block the secondary mirror. We compared the power levels obtained from these measurements with those obtained from the receiver's internal calibration targets and we obtained $n_{\text {int }}=0.90 \pm 0.01$.

Although the reflecting layer, $0.07 \mu \mathrm{m}$ of aluminum, on the optics is only $1 / 3$ skin depth at $183 \mathrm{GHz}$, calculations following Stratton (1941) predicted a reflectivity of 0.994 . We placed the loads so as to block the tertiary, and thereby determined that the reflection efficiency of the tertiary was $0.987 \pm 0.005$. We thus estimate that the efficiency due to reflection at all the optic surfaces, $n_{r}$, was $0.97 \pm 0.01$. 
Since our horn Gaussian beam pattern tapered to $-13 \mathrm{db}$ in the E-plane and $-10 \mathrm{db}$ in the $\mathrm{H}-\mathrm{plane}$ at the edge of the secondary, we calculate that $13-14 \%$ of the power reflected to the primary was subsequently blocked by the secondary $\left(n_{m o}=0.86-0.87\right)$. Thus, the telescope efficiency $n_{1}=$ $0.755 \pm 0.01$. We also calculated that $6-9 \%$ of the power spilled passed the secondary $\left(n_{\mathrm{fss}}=0.925 \pm 0.015\right)$.

Figure 2 gives $\tau_{a}(\nu)$ calculated using the model of Waters (1976). The profile shown is obtained from pressure broadening above $25 \mathrm{~km}$ altitude (Waters et al. 1980b). Absorption below $15 \mathrm{~km}$ altitude, in that region where some variability occurs in the water content of the atmosphere, contributes a uniform optical depth of $\sim 0.03$ across a band exceeding 500 $\mathrm{MHz}$. Therefore the calculated profile is, for practical purposes, constant for all conditions encountered at the KAO operating altitude. An interhemispheric survey found only small vartation in the $183 \mathrm{GHz}$ emission between latitudes of $+60^{\circ}$ and $-70^{\circ}$ (Waters et al. 1980b). The calculated average atmospheric opacity across our $100 \mathrm{MHz}$ bandwidth is 0.12 .

The receiver efficlency, $n_{1}$, and atmospheric transmission calculation can be tested against measurements of the atmospheric brightness temperature. The antenna temperature observed on "cold" sky can be expressed as

$$
T_{S K Y}=\left(1-\eta_{1}\right) T_{A M B}+\frac{\eta_{1} T_{A I R}}{2}\left[1-e^{-\tau_{a}\left(\nu_{S}\right) A}\right]+\left[1-e^{-\tau_{a}\left(\nu_{i}\right) A}\right]
$$

where $\eta_{1}=0.755 \pm 0.01 ; \mathrm{T}_{\mathrm{AMB}}$ is estimated, on the basis of various sensors in the telescope cavity, to be $260 \pm 10 \mathrm{~K}$; TAIR is estimated from the U. S. Standard Atmosphere (1976) to be $250 \pm 10 \mathrm{~K} ; \tau_{\mathrm{a}}\left(\nu_{\mathrm{S}}\right)$, the average atmospheric opacity in the signal sideband, is 0.12 ; and $\tau_{a}\left(\nu_{1}\right)$, the average opacity in the image sideband, is calculated to be 0.003 . Thus, we calculate that $\mathrm{T}_{\mathrm{SKY}}=78 \pm 4 \mathrm{~K}$ at an a1rmass of 1.4 . We measured the antenna temperature 
on the sky to be $79 \pm 2 \mathrm{~K}$ at that airmass. Comparable results had been obtained during the 1976 and 1977 flights, indicating that $n_{1}$ was the same, to within $5 \%$, for all the measurements.

The effictency factors $n_{f s s}$ (spillover) and $n_{c}$ (source coupling) were calculated assuming a Gaussian fleld distribution over the primary with no scattering at the reflecting surfaces. The haif power beamwith was calculated to be 7.9 arcmin in the E-plane and 7.6 arcmin in the H-plane. We computed the aperture efficiency, $n_{A}$ (which can be expressed as $n_{r} n_{i n t} n_{f s s} t i m e s$ the on-axis gain divided by the on-axis gain for an equivalent, unfformly 111 uminated, unobscured aperture), to be $0.47 \pm .02$.

Since there was no point source strong enough to make an observational verification practical, we made measurements of the moon. The brightness distribution of the moon at $1.64 \mathrm{~mm}$ was interpolated from models calculated by Keihm (1983) for 1.2 and $3.3 \mathrm{~mm}$, taking into account the orientation of the E-vector as projected on the moon. Figure. 3 shows the computed scan profile, normalized to the central disk antenna temperature, as well as the measured points. The coupling efficiency, $n_{c}$, for the central position was computed to be 0.88 , which is about $2 \%$ less than that computed for a uniformly bright moon, so that we believe that this efficiency may be accurate to about \pm 0.02 . The center disk brightness temperature interpolated from Keihm's models is $345 \pm 10 \mathrm{~K}$. The airmass for these observations was 1.4 , so that we calculate an antenna temperature difference with respect to cold sky of

$$
\begin{aligned}
\Delta \mathrm{T}_{\mathrm{A}} & =\mathrm{T}_{\text {moon }}-\mathrm{T}_{\text {sky }} \\
& =\eta_{1} \eta_{\text {fss }} \eta_{\mathrm{c}} \frac{(345 \pm 10 \mathrm{~K})}{2}\left[\mathrm{e}^{-\tau_{\mathrm{a}}\left(\nu_{\mathrm{s}}\right) \mathrm{A}}+\mathrm{e}^{-\tau_{\mathrm{a}}\left(\nu_{\mathrm{i}}\right) \mathrm{A}}\right] \\
& =195 \pm 8 \mathrm{~K}
\end{aligned}
$$


The measured antenna temperature at the moon's center was $165 \pm 3 \mathrm{~K}$. The difference between these is about twice our estimated uncertainty and represents an additional loss of $15 \%$ that we have not accounted for. Because the discrepancy is not seen in the sky brightness measurenent, we suspect our simplified beam pattern calculation. Factors which have been overlooked, because they were not convenient to incorporate into our method of calculation, include diffraction at the edge of the secondary, and obscuration and diffraction by the spider. Based on our actual measured antenna temperature on the moon, we therefore derive an overall efficiency of

$$
\left(n_{1} n_{\text {fss }} n_{c}\right)=0.52 \pm 0.05
$$

and also revise our 1980 aperture efficiency estimate downward by the same fraction to $n_{A}=0.40$

In 1976 October, the moon was measured to be $153 \pm 5 \mathrm{~K}$, corrected for atmospheric absorption, at the center of the illuminated portion for which a brightness temperature of $315 \pm 15 \mathrm{~K}$ was estimated (Waters et a1. 1980a). The lower overall efficiency $\left(n_{1} n_{\text {fss }} n_{c}\right)$ of $0.49 \pm 0.05$ is entirely accounted for by a stronger edge taper $(\sim 18 \mathrm{db})$ used to illuminate the secondary. We conclude that the efficiencies for extended sources agree for the two receivers to within $10 \%$.

One concern might be the effect of an error in our designed illumination of the secondary. Figure 4 shows the computed aperture and moon coupling efficiences including the empirical $15 \%$ correction. Based on the designed edge tapers, we would expect a 15-20\% stronger signal from an unresolved source in 1980 February than in 1977. Since the difference between the designed and actual edge tapers is, at most, one or two dB, we can rule this out as a possible cause for the apparent near doubling of the 
antenna temperature (and certainly would not explain the frequency shift). Furthermore, any errors in 111 umination so gross as to cause this effect would also be evident in the moon coupling efficiency.

\section{c) Results}

Figure 5 shows the observations made in 1977 December (Waters et al. 1980a) and 1980 February, smoothed to a resolution of $0.5 \mathrm{~km} \mathrm{~s}^{-1}$. The integration times were 110 minutes in 1977 and 19 minutes in 1980 . No baseline corrections have been applied. The single sideband temperature scale, which is twice the antenna temperature scale on the assumption that the gains in the two sidebands were equal, has been normallzed by dividing each data set by the appropriate aperture efficlency. The assumption of equal noise power in the sidebands is justified by the narrow sideband separation, $2.8 \mathrm{GHz}$, relative to the observing frequency and the fact that we always tuned to the backshort position closest to the diode.

The difference between the 1977 and 1980 observations cannot be attributed to polarization. The 1980 spectrum is an average of data taken on two consecutive nights. Differences in the hour angle at the time of observation caused the E-vectors to have position angles of $117^{\circ}$ and $170^{\circ}$, respectively. There were no differences greater than $2 \mathrm{~K}$ between the two separate observations. Comparison with spectra of other sources showed this to be within the noise of the digital spectrometer. If the radiation were $100 \% 1$ inearly polarized, these two observations could only be reconciled if the polarization vector lay in the position angle range of either $50: 5$ to $56^{\circ}$ or $133^{\circ}$ to $156^{\circ}$. The recelver E-vector position angle in 1977 December varied between $140^{\circ}$ and $165^{\circ}$, so that only the $50^{\circ}-56^{\circ}$ range of polarization angles could yield a weaker signal for the 1977 observations. A1so, because 
of variation in the position angle during the course of the observations, the intensity of the s!gnal, relative to the 1980 observations, should then have varied between at least $50 \%$ and $80 \%$, and possibly as much as 0 to $84 \%$, depending on the degree of polarization and the position angle of the polarization vector. In fact, no such variation was seen during the 1977 December observations.

The difference between 1977 and 1980 cannot be attributed to atmospheric effects. The vertical dotted line in Figure 5 represents the position of the terrestrial $\mathrm{H}_{2} \mathrm{O}$ line in 1977 December. Since the alrmass for these observations was 1.5 , the correction factor at $10 \mathrm{~km} \mathrm{~s}^{-1}$ for losses through the atmosphere is 1.19. In 1980 February, the terrestrial line was at $-39.5 \mathrm{~km} \mathrm{~s}^{-1}$ with respect to the local standard of rest; and the airmass was 1.6, giving a correction factor of 1.18 at $10 \mathrm{~km} \mathrm{~s}^{-1}$. The observations made in 1976 October and 1977 October (Waters et al. 1980a) agree to within the noise, when atmospheric losses are taken into account, with the 1977 December data.

A dashed fiducial line has been drawn through the peak of the 1977 spectrum, at $10 \mathrm{~km} \mathrm{~s}^{-1}$, to aid in comparing the spectra. The peak SSB antenna temperature in 1977 December was $7 \mathrm{~K}$. In 1980 February, the peak was $13 \mathrm{~K}$ and had shifted to $11.5 \mathrm{~km} \mathrm{~s}^{-1}$. Comparing the line wings, we see a dramatic increase in the range $15-20 \mathrm{~km} \mathrm{~s}^{-1}$, but only a little change in the range of $0-5 \mathrm{~km} \mathrm{~s}^{-1}$. The upper panel in Figure 5 contains the difference between and 1977 and 1980 spectra. The increase at $12 \mathrm{kms}^{-1}$ can be clearly seen. The peak intensity of the difference spectrum is comparable to that of the 1977 spectrum. The difference profile is narrower than the original spectrum, but still has broad wings which extend at least $15 \mathrm{~km} \mathrm{~s}^{-1}$ on elther side of the peak. 


\section{DISCUSSION}

The size of the high velocity source is somewhat dependent on the molecule, transition, and technique used to observe 1t (Table 1). The main HVS, at an LSR velocity of $\sim 9 \mathrm{~km} \mathrm{~s}^{-1}$, appears to be $\sim 20$ arcsec in diameter. If the $183 \mathrm{GHz}$ source were this size, the brightness temperature of the additional emission would be $\sim 5000 \mathrm{~K}$ near the inner part of the wings. If this emission arose in only part of the HVS, the brightness temperature would be proportionally higher. However, from the shape of the profile, we can infer that the emission region is at least large enough to include many individual clumps of HV gas.

In subsection a we present radiative transfer arguments which limit the brightness temperature. In subsection $b$ we consider the mechanisms which can pump the transition, which also argue against an extremely high brightness temperature. We then discuss, in subsection $c$, possible causes of the enhanced emission and, in subsection $d$, present the results of statistical equilibrium modelling which suggest an explanation for the phenomenon. In subsection e we discuss the relation between the $183 \mathrm{GHz}$ enhancement and the $22 \mathrm{GHz}$ flare.

a) Nature of the Emission

In spite of the change in the spectrum, which is usually an indication of maser action, the overall appearance suggests that there is little gain In the transition. For comparison, consider the $22 \mathrm{GHz} \mathrm{H}_{2} \mathrm{O}$ spectrum and the $18 \mathrm{~cm} \mathrm{OH} \mathrm{spectra} \mathrm{which} \mathrm{consist} \mathrm{of} \mathrm{numerous} \mathrm{spots} \mathrm{in} \mathrm{the} \mathrm{sky} \mathrm{emitting} \mathrm{in}$ very narrow bandwidths (typical $0.5-1 \mathrm{~km} \mathrm{~s}^{-1}$ ). If the rather smooth 183 $\mathrm{GHz}$ spectrum were to be composed of such features, there would need to be many more of them. But if the $183 \mathrm{GHz}$ transition should so easily go into 
a strong inversion, then $183 \mathrm{GHz}$ emission should be quite common in the Galaxy. Since it has been seen only in Orion (Waters et a1. 1980a), it appears that $183 \mathrm{GHz}$ emission is relatively difficult to produce. However, the conditions in Orion which favour the inversion of the transition are not very selective since the emission occurs without any small-scale structure in Doppler velocity and over a wide range of velocities. Since velocity coherence does not appear to be necessary to produce the emission, we conclude that amplification by stimulated emission does not play an important role.

Since the gain is small, the excitation temperature cannot also be small for a strong line to be seen. Thus, it follows that the population rat 10 must be near unity. With the assumptions of small optical depth and small population difference, the equation of transfer simplifies to

$$
T_{b}=3.77 \times 10^{-12} \frac{g_{u_{u}} n_{u}}{n_{\mathrm{H}_{2} \mathrm{O}}} \frac{\left[\mathrm{H}_{2} \mathrm{O}\right]}{\left[\mathrm{H}_{2}\right]} \frac{\mathrm{N}_{\mathrm{H}_{2}}}{\Delta V}
$$

where $n_{u}$ is the population per magnetic sublevel of the upper state in the the transition, ${ }^{\mathrm{H}_{2}} \mathrm{O}$ is the density of water molecules in all states, $\mathrm{g}_{\mathrm{u}}$ is the statistical weight of the upper level, $\mathrm{N}_{\mathrm{H}_{2}}$ is the gas column density, $\Delta \mathrm{V}$ is the linewidth in $\mathrm{km} \mathrm{s}^{-1}$, and the other symbols have their usual meanings. For illustration, we consider possible emission from the quiescent cloud which yields the spike feature. The gas column density is $2 \times 10^{23} \mathrm{~cm}^{-2}$ (Evans et al. 1975); the linewidth is $\sim 5 \mathrm{~km} \mathrm{~s}^{-1}$; and an upper 1imit to the population in the $3_{13}$ state may be given by $7 \exp (-204.5 / T) / Q$ where the partition function has been tabulated by Poynter and Pickett (1981). If we take $10^{-4}$ as an upper limit to the water abundance, we find that the brightness temperature $1 \mathrm{~s}<5 \times 10^{4} \mathrm{~K}$. If we assume that the water abundance is equal to the CO abundance (Iglesias and Silk 1978; Elitzur 
1979; Kuiper, Zuckerman, and Rodriguez Kuiper 1981) and estimate the CO abundance in orion to be $\sim 2 \times 10^{-6}$ (Wootten et a1. 1978), then we find that $\mathrm{T}_{\mathrm{B}} \ll 1000 \mathrm{~K}$. These crude estimates are consistent with the results of statistical equilibrium calculations and show, in a relatively stralghtforward way, that the $183 \mathrm{GHz}$ brightness temperature is quite modest compared to those inferred for strong masers (e.g., Reid and Moran 1981, Elitzur 1982).

The conditions in the high velocity region are not as well understood. The gas column density is significantly less because the CO $\mathrm{J}=0-1$ wings are not optically thick (Kuiper, Zuckerman, and Rodriguez Kuiper 1981). The abundance of water may be enhanced by shocks (Iglesias and Silk 1978). These two effects will tend to cancel each other, so that the conclustion that the brightness temperature in the $3_{13}-2_{02}$ transition must be modest, when compared to prominent astrophysical masers, still holds.

\section{b) Excitation of the Transition}

We first assess the role of collisions in populating the levels of the $3_{13}-2_{20}$ transition. Figure 6 shows the lower part of the energy level diagram for para- $\mathrm{H}_{2} \mathrm{O}$ with the allowed radiative transitions which connect these levels. The probability of spontaneous decay per second is shown next to the lines for the $\Delta K=1$ transitions. Collision rates have been calculated by Green (1980) to be in the range of $10^{-12}-10^{-11} \mathrm{~cm}^{3}$ $\sec ^{-1}$ for the $3_{13}$ and $2_{20}$ levels over a wide range of temperatures. Thus, gas densities on the order of $10^{10} \mathrm{~cm}^{-3}$ would be required in an optically thin cloud to excite the rotational levels collisionally. The far- $\mathrm{IR} \mathrm{H}_{2} \mathrm{O}$ transitions in Orion are, however, optically very thick. Both statistical equilibrium and LTE calculations yield estimates in the range of $10^{3}$ to $10^{4}$ 
for the extended, quiescent cloud with typical assumptions for temperature, density, column density, and water abundance. Consequently, the density requirement for collisional excitation is reduced to $\sim 10^{7} \mathrm{~cm}^{-3}$. In the case of clumps of hlgh velocity gas, denstites as high as $\sim 10^{7} \mathrm{~cm}^{-3}$ are plausible (Kuiper, Zuckerman, and Rodriguez Kuiper 1981; Masson et al. 1983). Although the role of collisions may not be entirely negligible, they cannot account for the observed high brightness temperature.

Following Goldreich and Kwan (1974), let us imagine that the 183 GHz population ratio is enhanced because of an excess of decays from the 220 level relative to the 313 level. (Indeed, the ratios of the appropriate probabilities for spontaneous decay, see Figure 6, makes this a plausible mechanism.) Therefore, for every $183 \mathrm{GHz}$ photon, there must be at least one photon at $2969 \mathrm{GHz}$. But since the $183 \mathrm{GHz}$ brightness temperature is only 10 to 100 times the excitation temperature of far-IR transition, the $183 \mathrm{GHz}$ emission is enhanced over spontaneous decay by only a factor of 10 to 100 . So, for every $183 \mathrm{GHz}$ photon, there must be approximately $10^{3-4} 3$ THz photons. Now the number of photons emltted per second at $183 \mathrm{GHz}$ per unit velocity interval is

$$
\begin{aligned}
\Delta \mathrm{N}_{\mathrm{m}} / \Delta \mathrm{V} & =4 \pi \mathrm{D}^{2}\left(\frac{\Omega_{\mathrm{m}}}{4 \mathrm{I}}\right) \cdot \mathrm{S} \cdot\left(\frac{\Delta \nu}{\mathrm{hv}}\right) / \Delta \mathrm{V} \\
& =10^{45} \mathrm{~T}_{\mathrm{A}} \quad\left(\mathrm{km}^{-1} \mathrm{~s}\right)
\end{aligned}
$$

where $D$ is the distance to Orion $(460 \mathrm{pc}), \Omega_{\mathrm{m}}$ is $4 \pi$ since the gain is small, $S=2 \mathrm{kT}_{\mathrm{A}} / \mathrm{n}_{\mathrm{A}} \mathrm{A}$, where $\mathrm{A}$ is the physical area of the telescope, and the other terms have their usual meanings. Thus, $\underset{\sim}{\sim} 10^{48}$ photons per second must be emitted by a clump at $3 \mathrm{THz}$. However, a blackbody at $100 \mathrm{~K}$ can only radiate $10^{45} \Theta^{2}$ photons per second at $3 \mathrm{THz}$, where $\theta$ is the angular radius of the source at the distance of Orion. Thus, only the entire HV source as seen in 
CO (Table 1) could emit enough far-IR radiation for this mechanism. While the emission rate per unit mass increases with decreasing clump size, a lot of small clumps in close proximity does not increase the overall loss rate from the ensemble because most clumps would radiate into each other. The efficiency of collisional pumping by the loss of far-IR line photons is enhanced by the presence of cold dust by a factor of the ratio of the dust optical depth to the line optical depth (Goldreich and Kwan 1974, 1979). Gatley et al. (1977) and Harvey, Campbell, and Hoffman (1979) find that the absorption efficiency of dust varies as $\lambda^{-1}$ from the near-IR to the far-IR. Using a ratio of $\mathrm{A}_{\mathrm{v}} / \mathrm{N}_{\mathrm{H}}=2 \times 10^{-21} \mathrm{mag} \mathrm{cm}^{-2}$, we find $\tau(102 \mu) \simeq 10^{-23} \mathrm{~N}_{\mathrm{H}_{2}}$. The optical depth in the $2_{20}-1_{11}$ transition is $1.8 \times 10^{-13} \mathrm{~N}_{\ell}\left(1-\mathrm{n}_{\mathrm{u}} / \mathrm{n}_{\ell}\right) / \Delta V$, where $\Delta V$ is $1 \mathrm{n} \mathrm{km} \mathrm{s}$. For an approximate evaluation, we assume that the levels are populated according to LTE so that $\tau\left(2_{20}-1_{11}\right) / \tau(102 \mu)$ is within a factor of two of $10^{9}\left(\mathrm{~N}_{\mathrm{H}_{2}} \mathrm{O} / \mathrm{N}_{\mathrm{H}_{2}}\right) / \Delta V$ for the temperature range $60-200 \mathrm{~K}$. For a predicted abundance of $10^{-6}$ for water, we the escape of photons from the cloud is by several orders of magnitude the dominant loss mechanism of $220-111$ photons.

Although the situation is more difficult to analyze for a collisional pump which overpopulates the 313 level, because of the large number of levels involved, the same order of magnitude conclusion applies. Also, as we discuss below, the variabiltty argues convincingly against a collisional pump.

Far-infrared radiation, however, does appear to be able to provide the necessary excitation. We can use the Einstein relations and the Planck distribution to write the ratio of the spontaneous decay rate to the rate of radiative excitation as 


$$
\frac{B A_{u 1}}{(1-\beta) B_{1 u}{ }^{W B}{ }_{\nu}\left(T_{1 R}\right)}=\frac{\beta}{(1-\beta) W}\left(\frac{g_{u}}{g_{1}}\right) \exp \left(\frac{\nu(G H z)}{20.8 T}-1\right)
$$

where $\beta$ is the probabllity that a photon will pass through the cloud without being reabsorbed, $W$ is a dilution faction which applies to the mean intensity of the exciting radiation, incorporating both optical depth and filling factor considerations, and the other symbols have their usual meanings. The frequencies of the principal transitions by which the 220 and 313 levels decay are 3 and $2.2 \mathrm{THz}$, respectively; so that for temperatures in the range of $100-150 \mathrm{~K}$, the quantity in square brackets is on the order of unity. If the escape probability in the far-IR transitions does not approach unity (see above), then the competition between spontaneous decay and radiative excitation depends principally on the ratio $\beta / W$. If we assume that the source of IR radiation and the emitting gas are generally in close proximity, then this ratio becomes the ratio of the far-IR optical depth in the dust to the optical depth in the far-IR transitions of $\mathrm{H}_{2} \mathrm{O}$. As noted before, $\tau(100 \mu) \simeq 10^{-23} \mathrm{~N}_{H_{2}}$. The optical depths in the $2_{20}-1_{11}$ and $3_{13}-2_{02}$ transitions are both $1.8 \times 10^{-13} N_{1}\left(1-n_{u} / n_{1}\right) / \Delta V$. Since the second term in the brackets becomes significant only after the upper levels have been populated, we may ignore it and note that only one water molecule for every $10^{10} / \Delta \mathrm{V}$ gas molecules need be in the lower rotational states for the $3_{13}$ and 220 levels to become populated by radiative excitation. If, as we previously estimated (Waters et al. 1980a) the abundance of water is $\sim 10^{-6}$, there seems to be no difficulty, for any reasonable velocity dispersion in a clump, in having enough opacity in the far-IR rotational lines of water for radiative excitation to be effective.

If, as we have estimated (Kuiper, Zuckerman, and Rodriguez Kuiper 1981), the abundance of water is higher in the HV gas, then FIR radiative 
excitation will be even more effective for the HV gas.

We can easily verify that a far-IR pump can produce enough emission by noting that there are sufficient pump photons to produce the observed number of $183 \mathrm{GHz}$ photons. On the assumption that the Doppler widths of the 183 $\mathrm{GHz}$ and the pump transition are the same and using the Rayleigh-Jeans approximation, one obtains for a saturated maser (e.g., Elitzur 1982)

$$
\mathrm{T}_{\mathrm{B}}(183 \mathrm{GHz})<140 \mathrm{~T}_{\mathrm{B}}(2164 \mathrm{GHz})\left(\Omega_{\mathrm{p}} / \Omega_{183}\right) \text {. }
$$

where $\Omega_{\mathrm{p}}$ is the solid angle subtended by the far-IR source at the $183 \mathrm{GHz}$ emitting region, and $\Omega_{183}$ is the solid angle into which the $183 \mathrm{GHz}$ emission is beamed. Since the dust and gas are co-extensive, and the 183 $\mathrm{GHz}$ transition radiates principally by spontaneous emission, these angles are both $4 \pi$. We consider first the extended cloud (i.e., "ridge") for which the parameters are better known. Averaged over the central arcminute, the emissivity of the dust at $200-300 \mu$ is $\sim 0.1$, and the temperature is $\sim 70 \mathrm{~K}\left(\mathrm{e} . \mathrm{g}\right.$. , Werner et al. 1976) so that $\mathrm{T}_{\mathrm{B}}(2164 \mathrm{GHz}) \sim 5-7 \mathrm{~K}$ and $\mathrm{T}_{\mathrm{B}}$ $(183 \mathrm{GHz})<1000 \mathrm{~K}$. For a low gain, unsaturated maser, the brightness temperature would be significantly less, which is consistent with the observations (Waters et al. 1980a). Thus, we conclude that the extended, quiescent molecular cloud will emit detectable $183 \mathrm{GHz}$ radiation and will have large optical depths in the far-IR water transitions, unless the abundance of water is an order of magnitude or more lower than predicted by chemical models. Within dense clumps of HV gas, the extinction can possibly be higher than the average over one arcminute. Thus, higher 183 $\mathrm{GHz}$ brightness temperatures may be expected for $\mathrm{HV}$ clumps. However, it is unlikely to be more than one or two orders of magnitude brighter. Consequent1y, the emission region would not be smaller than a few arcseconds. We note in passing, the near-IR excitation by hot dust is not likely 
to be an important mechanism. The model proposed by Goldreich and Kwan (1974) is quite general and can be applied with little modification to any transition in the ground vibrational state. It involves excitation to the first vibration state by means of $6 \mu \mathrm{m}$ radiation. The mechanism requires high density $\left(\sim 10^{9} \mathrm{~cm}^{-3}\right)$ and gas kinetic temperature $(\sim 1000 \mathrm{~K})$, and a dust temperature several hundred degrees above the gas temperature. However, the high velocity gas is much more rarified $\left(\stackrel{\sim}{\sim} 10^{7} \mathrm{~cm}^{-3}\right)$ and $\operatorname{cooler}(\sim 100 \mathrm{~K})$.

\section{c) Origin of the Emission}

We have previously argued that the high-velocity wings originate in clumps of gas from the prestellar molecular cloud which have been accelerated to high velocities by the wind of one or more newly formed stars (Kuiper, Zuckerman, and Rodriguez Kufper 1981). Since the dispersal of the original molecular cloud is yet in an early stage, the flow is channelled in a mostly random and turbulent way through the interstices which have formed in the cloud. Thus, the shape of the high-velocity wings does not come primarily from an accelerating radially outward flow, but from a high randomized flow which has a small net outward component. The significance of this model in the current context is that, at angular resolutions insufficient to resolve the individual clumps, subdivisions within the region of turbulent flow will have roughly the same shape wings and that variations in the mean rest velocity among the various subdivisions manifest themselves mainly as changes in the velocity of the line center. Random changes of position with velocity are seen in high resolution maps in So (Plambeck et al. 1982), v = 0 Sio (Wright et al. 1983) and $\mathrm{NH}_{3}$ (Pauls et al. 1982). We note that the extent of these sources at any velocity greatly exceeds any shift between centroids at different velocities (i.e., random motions dominate over 
systematic ones). The phenomenon also manifests itself in the different sizes of the HV sources as seen in various transitions (Table 1).

Even for a region as sma11 as $10^{\prime \prime}$ across, which corresponds to $7 \times 10^{11}$ $\mathrm{km}$, it is hard to inagine any process, other than radiation, which can cause a change in three years, since a velocity exceeding $2 \times 10^{4} \mathrm{~km} \mathrm{~s}^{-1}$ would be required. Thus, one posible explanation for the increased $183 \mathrm{GHz}$ emission is that a new region in the Orion complex has become illuminated by additional far-IR radiation.

We can imagine several ways in which the far-IR radiation can be increased. These include 1) a variable star deeply embedded in the cloud, ii) a flare associated with such a star, and iii) a break in the obscuring material surrounding such a star. To evaluate these possibilities, it is useful to consider the diffusion time for near-IR radiation, which is responsible for heating the dust. Using. Goldreich and Kwan's (1974) estimate for the photon mean free path, the diffusion time in years is

$$
\begin{aligned}
\tau_{\mathrm{dif}} \simeq \frac{3 \mathrm{r}^{2}}{4 \mathrm{Cl} \ell_{\mathrm{ph}}} & =8 \times 10^{-2}\left(\frac{\mathrm{r}}{10^{15} \mathrm{~cm}}\right)^{2}\left(\frac{\mathrm{n}_{\mathrm{H}_{2}}}{10^{10} \mathrm{~cm}^{-3}}\right) \\
& =4 \times 10^{-4}\left(\frac{\Theta}{\operatorname{arcsec}}\right)^{2}\left(\frac{\mathrm{n}_{2}}{10^{6} \mathrm{~cm}^{-3}}\right) .
\end{aligned}
$$

We see that at the densities associated with clumps $\left(\sim 10^{7} \mathrm{~cm}^{3}\right)$, the dust could be heated over the entire HV source in $1.6 \mathrm{y}$. This, of course, is a gross overestimate since we believe that the clumps sparsely fill a less dense region (Kuiper, Zuckerman, and Rodriguez Kuiper, 1981).

We consider first the possibility of a change in a dense circumstellar obscuring layer being responsible for the change. Yorke and Krügel (1977) have given a model for a dense protostellar cocoon with an inner radius of $10^{13} \mathrm{~cm}$ where the gas density is $10^{12} \mathrm{~cm}^{-3}$. The density varies roughly as 
$\mathrm{r}^{-2}$. Using the above formula, we see that the radiation takes $\sim 10^{-3}$ y to diffuse out through $10^{13} \mathrm{~cm}$, and thereafter doubles $1 \mathrm{ts}$ distance from the center every $10^{-3} \mathrm{y}$. Actually, this is an overestimate because as the radiation moves outward, the bulk of the energy shifts to longer wavelengths, so that the photon mean free path gets longer. Anyway, in about four days, the radiation has diffused out $10^{17} \mathrm{~cm}$, where the density is $\sim 10^{6} \mathrm{~cm}^{-3}$. Except in the unlikely case that the density of a cocoon is several orders of magnitude higher, the circumstellar dust cannot delay the IR radiation long enough to make a physical change in the cocoon a significant event in the heating of the outer layers.

Consequent $1 y$, any change in the IR radiation must be related to changes at the energy source(s). However, the increased $183 \mathrm{GHz}$ emission cannot be due merely to an increase in the far-IR radiation which pumps the transition. In that case, there would have to be a proportional increase in the far-IR luminosity. Gehrz et al. (1983) have monitored the Orion KL region at wavelengths between 2 and $20 \mu \mathrm{m}$ and have not found any variations larger than $30 \%$. Now it is clear from the change in the shape of the profile, as well as the probable flare nature of the causal event, that only part of the $\mathrm{HV}$ region is involved in the change. Thus, a $183 \mathrm{GHz}$ increase due solely to increased far-IR pumping, confined to a part of the HV source, would require the far-IR increase to be locally even larger, by a factor which is the inverse of the ratio of the area of the affected region to the entire HV source. Since the amount of dust, and hence the far-IR opacity, can be considered constant, a several-fold far-IR flux increase could only be due to an increase of the dust temperature to several hundred Kelvin, which would certainly be evident near $20 \mu$. Also we would expect the dust to cool promptly on a time scale given by Equation 7, which provides the 
physical basis for the mechanism identifled by our modelling calculations.

\section{d) Statistical Equilibrium Modelling}

We have carried out statistical equilibrium calculations with photon trapping to determine the conditions 1 ikely to cause a major increase in $183 \mathrm{GHz}$ emission. These calculations were carried out using the large velocity gradient (LVG) hypothesis so that radiative coupling between distant parts of a cloud can be 1gnored. In our model, the decoupling arises from the large relative separation of the clumps of HV gas as well as differential velocities between the clumps. It is not meaningful in this case to use an explicit velocity gradient formulation, $\mathrm{n}_{\mathrm{H}_{2}}(\mathrm{dV} / \mathrm{d} \ell)^{-1}$. Instead, we consider explicitly the column density $\mathrm{n}_{\mathrm{H}_{2}} \mathrm{~d} l$, and the velocity dispersion, $\Delta \mathrm{V}$, in the clumps. Sobolev (1957) and Whtte (1977) have suggested that this is a reasonable approximation for clouds without velocity gradients. We sinplified our calculations by neglecting absorption by dust as being negligible relative to line absorption (see above), and saturation (i.e., the escape probability was forced to be less than or equal to one) because high gain cases are not relevant to the present observations. We used the collision cross sections computed by Green (1980) for the ten lowest rotational levels of para- $\mathrm{H}_{2} \mathrm{O}$. Because the $4_{22}-3_{13}$ transition is one of the major pathways feeding the upper level of the $183 \mathrm{GHz}$ transition, we extended the published cross sections with unpublished estimates by Green (1979) for the rates connecting the next three levels. (The levels 431 and 515 are not shown in Figure 6.) Following white (1977), we expect that the numerical results are correct to within about half an order of magnitude. The radiation intensity was taken to be blackbody shortward of the wavelength, $\lambda_{c}$, at which the optical depth of the dust is unfty. Longward, 
the intensity was taken as blackbody multiplied by an emissivity which was inversely proportional to the wavelength (recall subsection b). It should be noted that the quantitative (but not the qualitative) results are sensitive to the wavelength dependence of the dust emissivity. Using the previously discussed ratio of IR optical depth to total gas column density, we computed cases for $\mathrm{N}_{\mathrm{H}_{2}}=5 \times 10^{21}, 10^{22}, 2.5 \times 10^{22}, 5 \times 10^{22}$ and $10^{23}$ $\mathrm{cm}^{-2}$, corresponding to $\lambda_{c}=5,10,25,50$, and $100 \mu \mathrm{m}$, respectively. For each column density, we computed three cases: gas and dust at $80 \mathrm{~K}$, gas at $80 \mathrm{~K}$ and dust at $200 \mathrm{~K}$, and gas at $200 \mathrm{~K}$ with dust at $80 \mathrm{~K}$. (A temperature of $\sim 200 \mathrm{~K}$ is suggested by the excitation temperature of ammonia in the "hot core" source-Morris, Palmer, and Zuckerman 1980; Ziurys et al. 1981.) In each instance, we carried out the calculations for densities from $10^{4}$ to $10^{9} \mathrm{~cm}^{-3}$ by steps of factors of ten.

We found that, in all cases, a density of $10^{9} \mathrm{~cm}^{-3}$ was sufficient to thermalize the $3_{13}-2_{20}$ level. With both the gas and dust at $80 \mathrm{~K}$, the strength of the $183 \mathrm{GHz}$ inversion decreased with increasing column density and, in all cases, was most prominent for a density of $10^{6} \mathrm{~cm}^{-3}$. Brightness temperatures significantly in excess of $100 \mathrm{~K}$ required that $\mathrm{N}_{\mathrm{H}_{2}} \mathrm{O} / \Delta \mathrm{V}>1017$ $\mathrm{cm}^{-2} \mathrm{~km}^{-1} \mathrm{~s}$ or a relative abundance of water on the order of $10^{-5}$ or higher. Increasing the temperature of the dust to $200 \mathrm{~K}$ largely suppressed the inversion. In this case, the brightest temperatures were $\sim 1000 \mathrm{~K}$ and required $\mathrm{N}_{\mathrm{H}_{2}} \mathrm{O} / \Delta \mathrm{V} \sim 10^{18} \mathrm{~cm}^{-2} \mathrm{~km}^{-1} \mathrm{~s}$, corresponding to a relative water abundance of $\sim 10^{-4}$. On the other hand, Increasing the temperature of the gas to $200 \mathrm{~K}$ (with the dust at $80 \mathrm{~K}$ ) generally increased the brightness temperatures. Indeed, if the water abundance is high enough $\left(\stackrel{>}{\sim}\right.$ ew $\left.\times 10^{-5}\right)$, it appears that very strong maser emission can occur. Increases as high as a factor of 50 were obtained for more modest relative abundances $\left(<10^{-5}\right)$. 
For example, at a total gas column density of $2.5 \times 10^{22} \mathrm{~cm}^{-2}$, a relative water abundance of $2 \times 10^{-6}$, a local 1 ine width of $1 \mathrm{~km} \mathrm{~s}^{-1}$, and a density of $10^{7}$ (i.e., a source size of $2.5 \times 10^{15} \mathrm{~cm}$ or $\sim 0.4 \mathrm{arcsec}$ ) the brightness temperature is $80 \mathrm{~K}$ for a gas temperature of $80 \mathrm{~K}$, and $800 \mathrm{~K}$ for a gas temperature of $200 \mathrm{~K}$. At a density of $10^{6}$ (i.e., a source size of $\sim 4$ arcsec) and a relative abundance of $4 \times 10^{-6}$, these numbers are $100 \mathrm{~K}$ and $1000 \mathrm{~K}$, respectively. These numbers should be taken only to illustrate the qualitative result that an increase in the gas temperature can strongly brighten the $183 \mathrm{GHz}$ emission.

We conclude, therefore, that the observed increase in the $183 \mathrm{GHz}$ emission was most 1 ikely due to an increase in the temperature of the gas.

e) Relation to the $22 \mathrm{GHz}$ Flare

In 1979 0ctober, a $22 \mathrm{GHz} \mathrm{H}_{2} \mathrm{O}$ maser feature at $8 \mathrm{~km} \mathrm{~s}^{-1} \mathrm{flared}$ to reach a peak of $>10^{6} \mathrm{Jy}$ (Matveyenko et a1. 1980; Abraham et al. 1981). The outburst decayed more or less stead1ly until the middle of 1980 February, at which time it was still more than an order of magnitude stronger than any previously observed water maser feature in orion. Late in February, the feature flared again, twlce as strong as before, and then decayed to a level of $\sim 10^{6} \mathrm{Jy}$ where it has remained since, varying by $\sim 20 \%$ (Garay, Moran, and Haschick 1983). The general time signature of such events has been shown to be consistent with an impulsive injection of energy (Burke, Giuffrida, and Haschick 1978). Evidence for impulsive release of mechanical energy assoctated with pre-main-sequence stars is deduced by Mund and llartman (1983) from the acceleration of $\mathrm{HH} 1$ and $\mathrm{HH} 2$, and from the occurrence of highly collimated jets (Graham and Elias 1983; Mundt and Fried 1983). The $22 \mathrm{GHz}$ flare occurred at a velocity which is in the wing of the 
$183 \mathrm{GHz}$ enhancement, not the velocity at which the $183 \mathrm{GHz}$ ine is the strongest. Also, we should bear in mind that the conditions which are believed to be present in $22 \mathrm{GHz}$ regions would thermalize the $183 \mathrm{GHz}$ transition. The $22 \mathrm{GHz}$ flare region could, however, come from a single dense clump in the $183 \mathrm{GHz}$ emission region. This region probably encompasses many other $22 \mathrm{GHz}$ masers (Genzel et al. 1981) which have not changed in a correlated manner (Cohen and Zisk, 1980). Consequently, any IR radiation associated with the $22 \mathrm{GHz}$ flare would have to be at wavelengths short enough to be effectively shielded by dust, say, a few microns or less. A possible relation between the events at $22 \mathrm{GHz}$ and $183 \mathrm{GHz}$ may be less direct than this. For example, mechanical energy associated with a flare event could have caused shock heating or possibly a favourable alignment of material which, together with IR radiation, could be responsible for the $22 \mathrm{GHz}$ maser (Abraham et al. 1981). The $183 \mathrm{GHz}$ enhancement would then be a secondary manifestation of the same flare event in the form of a temporary heating of the dust. In a model proposed by Deguchi (1981), a burst of infrared luminosity occurs at the center of an optically thick, cold, dusty cloud. (In principle, shock heating would serve the model equally we11.) Once the energy source is turned off, the dust cools more rapidly than the gas because the timescales for cooling the gas by radiation or collisions with the dust are much longer than the time for radiant energy from the dust to diffuse out of the cloud. A hot-gas, cold-dust pump, which also selectively removes $5_{23}-4_{14}$ photons via an absorption band at $43 \mu \mathrm{m}$, then produces the $22 \mathrm{GHz}$ maser. The $22 \mathrm{GHz}$ emission decays in a time set by the rate at which the gas molecules collide with the dust which, depending on the density, can be weeks to months (e.g., Goldreich and Kwan 1974, Deguchi 1981). 
The region heated can be considerably larger than the dense $\left(\sim 10^{9}-\right.$ $10^{10} \mathrm{~cm}^{-2}$ ) region giving rise to $22 \mathrm{GHz}$ emission. Even at a density of $10^{7} \mathrm{~cm}^{-3}$, a mean free path at $100 \mu$ is only $10^{16} \mathrm{~cm}(\sim 1.5$ arcsec $)$, so that the photons are considerably more likely to be absorbed than to escape. Thus, one would expect, as a natural consequence of Deguchi's model, an enhancement of the $183 \mathrm{GHz}$ emission over a larger region.

There is little difficulty in obtalning enough energy to do the heating. To heat the entire plateau source, estimated to be $\sim 3 \mathrm{M}_{\odot}$ (Zuckerman, Kuiper, and Rodriguez Kuiper 1976), from 80K to, say, $200 \mathrm{~K}$ would require $7.5 \times 10^{43}$ ergs. Since we require only a small part of the plateau region to be heated, let us put the energy requirement to heat the gas at $\sim 10^{43}$ ergs which is only $10^{-4}$ of all the mechanical energy present in the plateau (e.g., Bally and Lada 1983). If an impulsive event of this kind occurred every twenty years (22 GHz emisston was first observed in 1968 by Cheung et a1. 1969), then the average power required is $\sim 5 \mathrm{~L} \odot$ which is $\sim 10^{-4}$ of the bolometric luminosity of the Orion BN-KL complex, and $\sim 2 \times 10^{-3}$ of the mechanical luminosity in the high velocity flow.

At the densities most favourable to strong $183 \mathrm{GHz}$ emission $\left(10^{6-7}\right.$ $\mathrm{cm}^{-3}$ ), the time scale for the heated gas to lose its energy to the cold dust is $20-200 \mathrm{y}$ (Goldreich and Kwan 1974). If outburst of this kind occur as often as every few decades, one might possibly still see evidence of previous outbursts. VLA observations of $\mathrm{N}_{\mathrm{H}_{3}}$ shows most of the hot gas to be concentrated in four condensations within a $\sim 10^{\prime \prime}$ diameter region near the center of the Orion IR complex (Pauls et a1. 1983). These condensations are not responsible for the $183 \mathrm{GHz}$ enhancement or the $22 \mathrm{GHz}$ outburst because of discrepancles between the LRS velocities. Also, the Hot Core was recognized in observations as early as 1977 (Morris, Palmer, and Zuckerman 
1980). Townes et al. (1983), who observed the $a(4,3)-s(3,3)$ transition of $\mathrm{NH}_{3}$ at $125 \mu \mathrm{m}$ from the hot core, point out however that the gas is at least several tens of degrees hotter than the associated dust. Since only one of these condensations is associated with a known infrared source (IRc 4) and it is questionable whether that one has an internal energy source (Werner et al. 1983), the gas will eventually lose 1 ts energy by collisions with the dust unless it is being continuously resupplied with energy. Thus, it is concelvable that these hot spots are earlier manifestations of the kind of event which we infer to have occurred in 1979.

On a speculative note, if the flare event seen at 22 and $183 \mathrm{GHz}$ is a by-product of a kinetic event which released considerably more energy than is manifested in the heating of gas, then it is possible that such sporadic outbursts provide the energy and momentum for the high velocity gas.

\section{CONCLUSIONS}

The peak flux of the $183 \mathrm{GHz}$ water line emission from Orion increased by $50 \%$ between 1977 December and 1980 February. An increase was also seen in the wide wings of the 11ne. The profile shifted approximately $1.5 \mathrm{~km} \mathrm{~s}^{-1}$ to the red. We considered instrumental effects, absorption in the earth's atmosphere, and polarization effects as possible causes for the observed increase and have been unable to identify any sources of systematic error. Simultaneous, undetected errors in both the frequency and intensity calibrations seem to us very unlikely.

Because of the change in the shape of the profile, we concluded that the additional emission came from a subset of the entire high velocity ("plateau") source. The wide range of velocities over which the increase was seen suggests to us that it is not due to a high gain maser, but the 
inferred brightness temperatures are high enough to require low-gain maser action. Collisions do not appear to be capable of providing sufficient excitation in HV clumps because they cannot rid themselves of far-IR photons fast enough to support the pump. On the other hand, there is enough far-IR emission from the dust to excite the rotational ladder of water and produce the observed brightness temperatures. We carrled out statistical equilibrium calculations which indicate that the likely cause of a large increase of the $183 \mathrm{GHz}$ emission is impulsive heating of the gas and dust, but with the dust having quickly returned to approximately its original temperature.

The occurrence of a major flare in the $22 \mathrm{GHz}$ emission during the period of the $183 \mathrm{GHz}$ brightening suggests a common cause. Because of the high velocity of propagation which would be required for mechanical (shock) heating of the $183 \mathrm{GHz}$ region, a radiant heating seems likely, although the ultimate cause of the radiation may still be a a mechanical event. The energy required to cause the heating is only $10^{-4}$ of all the mechanical energy in the HV source. Even if such an event occurred only every few decades, the average power required is $\sim 10^{-4}$ of the bolometric luminosity and $\sim 2 \times 10^{-3}$ of the mechanical luminosity in the orion BN-KL complex. Separate evidence for hot gas in the presence of cool dust is found in the hot core source, studied principally in ammonia. The components of this source may be remnants of earlier flare events. The timescale for cooling of the gas responsible for $183 \mathrm{GHz}$ emission by collisions with the dust is expected to be in the range of 20 to 200 years. We also point out that if the heating of the gas, which is the proposed cause of the $183 \mathrm{GHz} f$ lare, is a by-product of a causative kinetic outburst, then the energy and momentum in the outburst might be large enough so that a succession of outbursts could be the driving force for the high velocity gas. 
We express our gratitude to P. Batelaan and W. Ricketts for the engineering support which made this work possible and to the staff of the G. P. Kuiper Airborne Observatory for their unstinting support of the observations. We also wish to thank J. Moran for access to an early draft of the paper by Garay, Moran, and Haschick (1983) and acknowledge helpful discussions with M. Frerking, R. Gehrz, S. Gulkis, M. Litvak, M. Malkan, J. Moran, D. Matsakis, P. Schwartz, and J. Waters. The research described in this paper was performed at the Jet Propulsion Laboratory, California Institute of Technology, under contract with the National Aeronautics and Space Administration. 
Table 1 - Sizes of HV Source in Various Transitions

\begin{tabular}{|c|c|c|c|c|}
\hline Molecule & Transition & $\frac{\text { Size }}{\operatorname{arcsec}}$ & $\frac{v_{1}}{k m} r_{s}-1$ & Reference \\
\hline $\mathrm{H}_{2} \mathrm{O}$ & $6_{1,6}-5_{2,3}$ & $30^{\prime \prime} \times 45^{\prime \prime}$ & $\sim 10$ & Genze1 et a1. 1981 \\
\hline \multirow[t]{5}{*}{$\mathrm{CO}$} & $1-0$ & $30-40$ & 9.5 & Solomon et a1. 1981 \\
\hline & $1-0$ & $\sim 20 "$ & 9 & Masson et a1. 1983 \\
\hline & & $\sim 11 "$ & 5 & Masson et al. 1983 \\
\hline & $2-1$ & $\sim 25^{\prime \prime}$ & 9 & Knapp et a1. 1981 \\
\hline & $3-2$ & $30-40 "$ & 9 & Erickson et al. 1982 \\
\hline $\mathrm{HCO}^{+}$ & $1-0$ & $<30^{\circ}(1)$ & $\sim 9$ & Olofsson et al. 1983 \\
\hline \multirow[t]{2}{*}{$\mathrm{SO}_{2}$} & $8_{1,7}-8_{0,8}$ & $\sim 25 "$ & 8.3 & Schloerb et al. 1983 \\
\hline & $131,13-120,12$ & $\sim 25 "$ & 9 & Knapp et a1. 1981 \\
\hline so & $2_{1}-1_{1}$ & $11 " \times 9 "$ & & Plambeck et a1. 1982 \\
\hline \multirow{3}{*}{ SiO } & $(3,3)$ & $10^{\prime \prime} \times 7 "$ & $\sim 5$ & $\begin{array}{l}\text { Bastein et al. } 1981 \\
\text { Pauls et a1. } 1982\end{array}$ \\
\hline & $1-0, v=0$ & $<10^{\prime \prime}$ & $\sim 5$ & Genzel et al. 1980 \\
\hline & $2-1, v=0$ & $15^{\prime \prime} \times 30 "$ & $5-9$ & Wright et a1. 1983 \\
\hline
\end{tabular}

(1) With a medium velocity feature extending $100^{\prime \prime}$ to the north. 


\section{REFERENCES}

Abraham, Z., Cohen, N. L., Opher, R., Raffaelli, J. C., and Zisk, S. H. 1981, Astron. Astroph., 100, L10.

Ba11y, J., and Lada, C. J. 1983, Ap. J., 265, 824. Bastien, P., Bleging, J., Henke1, C., Martin, R. N., Pauls, T., Walnsley, C. M., Wilson, T. L., and Ziurys, L. M. 1981, Astr. Astroph., 98, L4. Burke, B. F., Giuffrida, T. S., and Haschick, A. D. 1978, Ap. J., 226, L21. Cheung, A. C., Rank, D. M., Townes, C. H., Thornton, D. D., and Welch, W. J. 1969, Nature, 221, 626 .

Cohen, N. L., and Zisk, S. H. 1980, B. A. A. S., 12, 507.

Deguchi, S. 1981, Ap. J., 249, 145 .

E1itzur, M. 1979, Ap. J., 229, 560.

E1itzur, M. 1982, Rev. Mod. Phys., 54, 1225.

Erickson, N. R., Goldsmith, P. F., Snell, R. L., Berson, R. L., Huguenin, G. R., U1ich, B. L., and Lada, C. J. 1982, Ap. J., 261, L103.

Evans, N. J., II, Zuckerman, B., Sato, T., and Morris, G. 1975, Ap. J., $199,383$.

Garay, G., Moran, J. M., and Haschick, A. D. 1983, in preparation. Gatley, I., Becklin, E. E., Werner, M. W., and Wynn-Williams, C. G. 1977, Ap. J., $216,277$.

Genzel, R., Downes, D., Schwartz, P. R., Spencer, J. H., Pankonin V., and Baars, J. W. M. 1980, Ap. J., 239, 519.

Genze1, R., Reid, M. J., Moran, J. M., and Downes, D. 1981, Ap. J., 244, 884. Gehrz, R. Caldwe11, K., Price, J., Hackwe11, J., and Grasdalen, G. 1983, in preparation.

Goldrefch, P., and Kwan, S. 1974, AP. J., 191, 93.

Goldreich, P., and Kwan, S. 1979, Ap. J., 227, 150. 
Graham, J. A. and E11as, J. H. 1983, Ap. J., 272, 615.

Green, S. 1979, private communication.

Green, S. 1980, Ap. J. Supp1., 42, 103.

Harvey, P. M., Campbe11, M. F., and Hoffman, W. F. 1979, Ap. J., 228, 445.

Iglesias, E. R., and Silk, J. 1978, Ap. J., 226, 851 .

Keihm, S. 1983, private communication.

Knapp, G. R॰, Phillips, T. G., Huggins, P. J., and Redman, R. 0. 1981,

$$
\text { Ap. J., } 250,175 \text {. }
$$

Kuiper, T. B. H., Swanson, P. N., Dickinson, D. F., Rodriguez Kuiper,

E. N., and Zimmermann, P. 1984, in preparation.

Kuiper, T. B. H., Zuckerman, B., and Rodriguez Kuiper, E. N. 1981, Ap. J., 251,88 .

Kutner, M. L., and Ulich, B. L. 1981, Ap. J., 250, 341.

Masson, C. R., Berge, G. L., Claussen, M. J., Helligman, G. M., Leighton, R. B., Lo, K. Y., Moffet, A. T., Phillips, T. G., Sargent, A. I., Scott, S. L., Wannier, P. G., Woody, D. P. 1983, preprint.

Matveyenko, L., Kogan, L., and Kostenko, K. 1980, Sov. Astron.Lett., 6, 279. Morris, M., Palmer, P., and Zuckerman, B. 1980, Ap. J., 237, 1.

Mundt, R., and Fried, J. W. 1983, Ap. J. (Letters), 274, L83.

Mundt, R., and Hartmann, L. 1983, Ap. J., 268, 766.

Olofsson, H., Ellder, J., Hjalmarson, A., and Rydbeck, G. 1982, preprint.

Pauls, T. A., Wilson, T. L., Bieging, J. H., and Martin, R. N. 1983, Astr. Astrop., $124,23$.

Phil11ps, T. G., Kwan J., and Hugg1ngs, P. J. 1980, in Interstellar

Molecules (B. H. Andrews, ed.), Reidel, Dordrecht, p. 21.

Plambeck, R. L., Wright, M. C. H., Welch, W. J., Bieging, J. H., Baud, B., Ho, P. T. P., and Vogel, S. N. 1982, Ap. J., 259, 617. 
Reid, M. J., and Moran, J. M. 1981, A. R. A. A., 19, 231.

Schloerb, F., Friberg, P., Hjalmarson, A., Hoglund, B., and Irvine, W. M. 1983, Ap. J., 264, 161 .

Sobolev, V. V. 1957, Soviet Astronomy AJ, 1, 678.

Solomon, P. M., Huguenin, G. R., and Scoville, N. 2. 1981, Ap. J., 245, L19.

Stratton, J. A. 1941, Electromagnet ic Theory, McGraw-Hi11, New York,

p. $301 \mathrm{ff}$.

Townes, C. H. Genzel, R., Watson, D. M., and Storey, J. W. V. 1983, Ap. J. (Letters), 269, L11.

U. S. Standard Atmosphere, 1976, U. S. Gov't. Printing office, NOAA-S/T 76-1562, Washington, DC.

Waters, J. W. 1976, in Methods of Experimental Physics, Vo1. 12: Astrophysics, Part B: Radio Telescopes, Academic Press, New York, p. 142. Waters, J. W., Gustincic, J. J., Kakar, R. K., Kufper, T. B. H., Roscoe, H. K., Swanson, P. N., Rodriguez Kulper, E. N., Kerr A. R., and Thaddeus, P. 1980a, Ap. J., 235, 57.

Waters, J. W., Gustincic, J. J., Swanson, P. N., and Kerr, A. R. 1980b, in Atmospheric Water Vapour, Academic Press, New York, p. 229. Werner, M. W., Gatley, I., Harper, D. A., Becklin, E. E., Loewenstein, R. F., Telesco, C. M., and Thomson, H. A. 1976, Ap. J., 204, 420. Werner, M. W., DinersteIn, H. L., and Capps, R. W. 1983, Ap. J., 265, L13. White, R. E. 1977, Ap. J., 211,744 . Wootten, A., Evans, N. J., II, Snel1, R., and Vanden Bout, P. 1978, Ap. J. (Letters), L143.

Wright, M. C. H., Plambeck, R. L., Vogel, S. N., Ho, P. T. P., and Welch, W. J. 1983, Ap. J.. 267, L41. Yorke, H. W., and Kruge1, E. 1977, Astron. Astroph., 54, 183. 
Ziurys, L. M., Martin, R. N., Pauls, T. A., and Wilson, T. L. 1981, Astron. Astroph., 104,228 .

Zuckerman, B., Kuiper, T. B. H., and Rodriguez Kuiper, E. N. 1976, Ap. J., 209 , 2137.

Zuckerman, B., and Palmer, P. 1975, Ap. J. (Letters), 199, L35. 


\section{FIGURE CAPTIONS}

Figure 1 - Schematic diagram of receiver and telescope system for the 1980 August flights. As noted in the text, there was a minor difference in the method of calibration during the 1980 February flights.

Figure 2 - Optical depth through the atmosphere towards the zenith as a function of frequency relative to $183310.09 \mathrm{MHz}$. The horizontal line representing a range of Doppler velocities from -50 to +50 $\mathrm{km} \mathrm{s} \mathrm{s}^{-1}$ is provided to facilitate comparison with the Orion spectra in Fig. 3 .

Figure 3 - Measurements of a scan across the moon's equator are shown along with the results of convolving our calculated beam profile with a model of the lunar brightness temperature at $1.6 \mathrm{~mm}$, as discussed in the text.

Figure 4 - Aperture efficiency and moon coupling efficiency as a function of the illumination at the edge of the secondary reflector (edge taper). These efficiencies include the empirical 15\% correction discussed in the text.

Figure 5 - Observations of the $313-220$ transition of water in the direction of the Orion Nebula in 1977 December and 1980 February. It is assumed that the SSB antenna temperature is twice the observed (DSB) antenna temperature.

Figure 6 - The lower part of the para- $\mathrm{H}_{2} \mathrm{O}$ rotational energy ladder, showing the allowed transitions which connect the levels, and the Einstein A coefficients (probability of spontaneous decay per second) for the $\Delta K=1$ transitions. 
ADDRESSES OF THE AUTHORS

D. F. Dickinson - Lockheed Palo Alto Research Laboratory

3251 Hanover Street

Palo Alto, CA 94304

M. J. Klein, T. B. H. Kulper, P. N. Swanson, P. Z1mmermann

Jet Propulsion Laboratory

California Institute of Technology

4800 Oak Grove Drive

Pasadena, CA 91109

E. N. Rodriguez Kuiper - Bennett Medical Equipment

12655 Beatrice Street

Los Angeles, CA 90066 


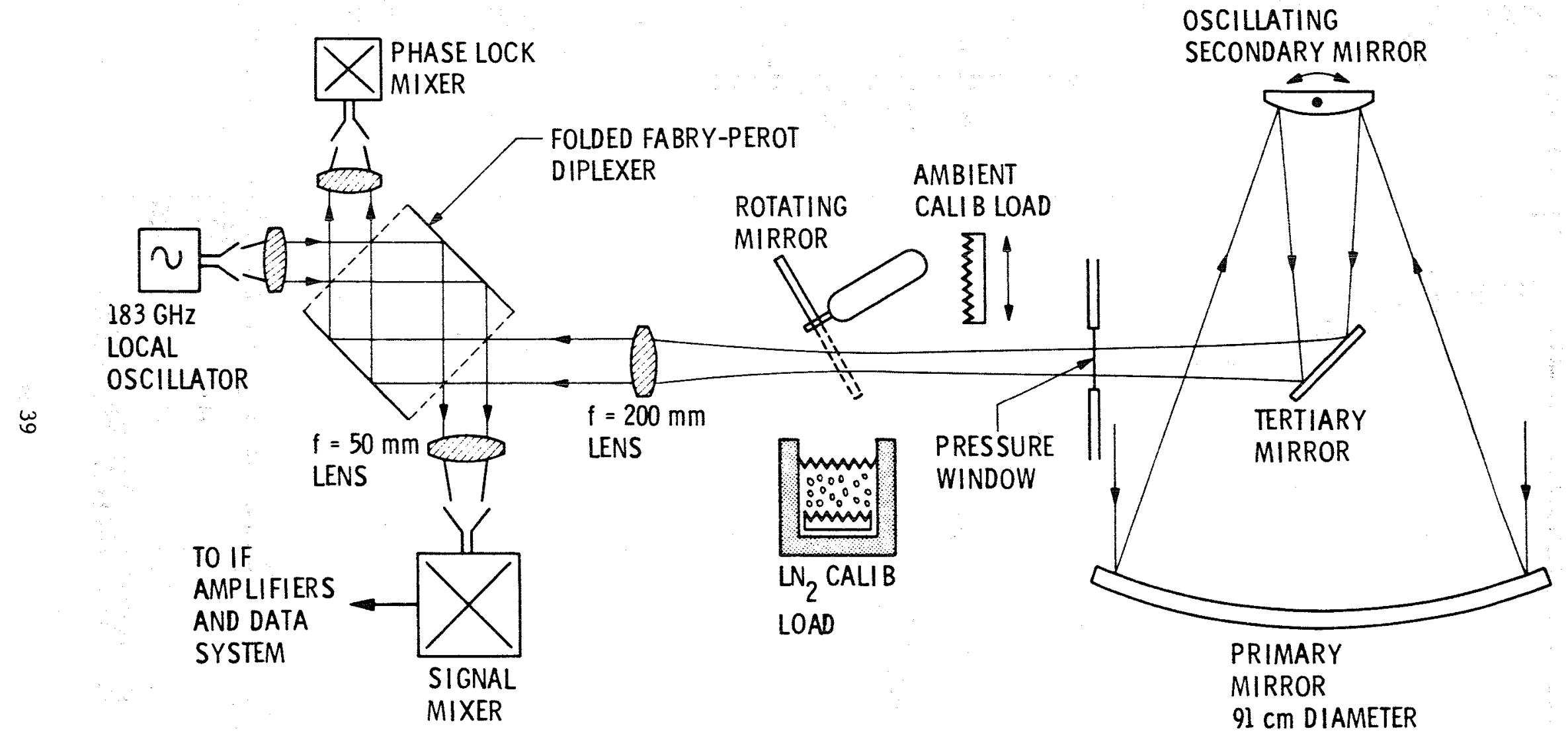

Fig. 1 


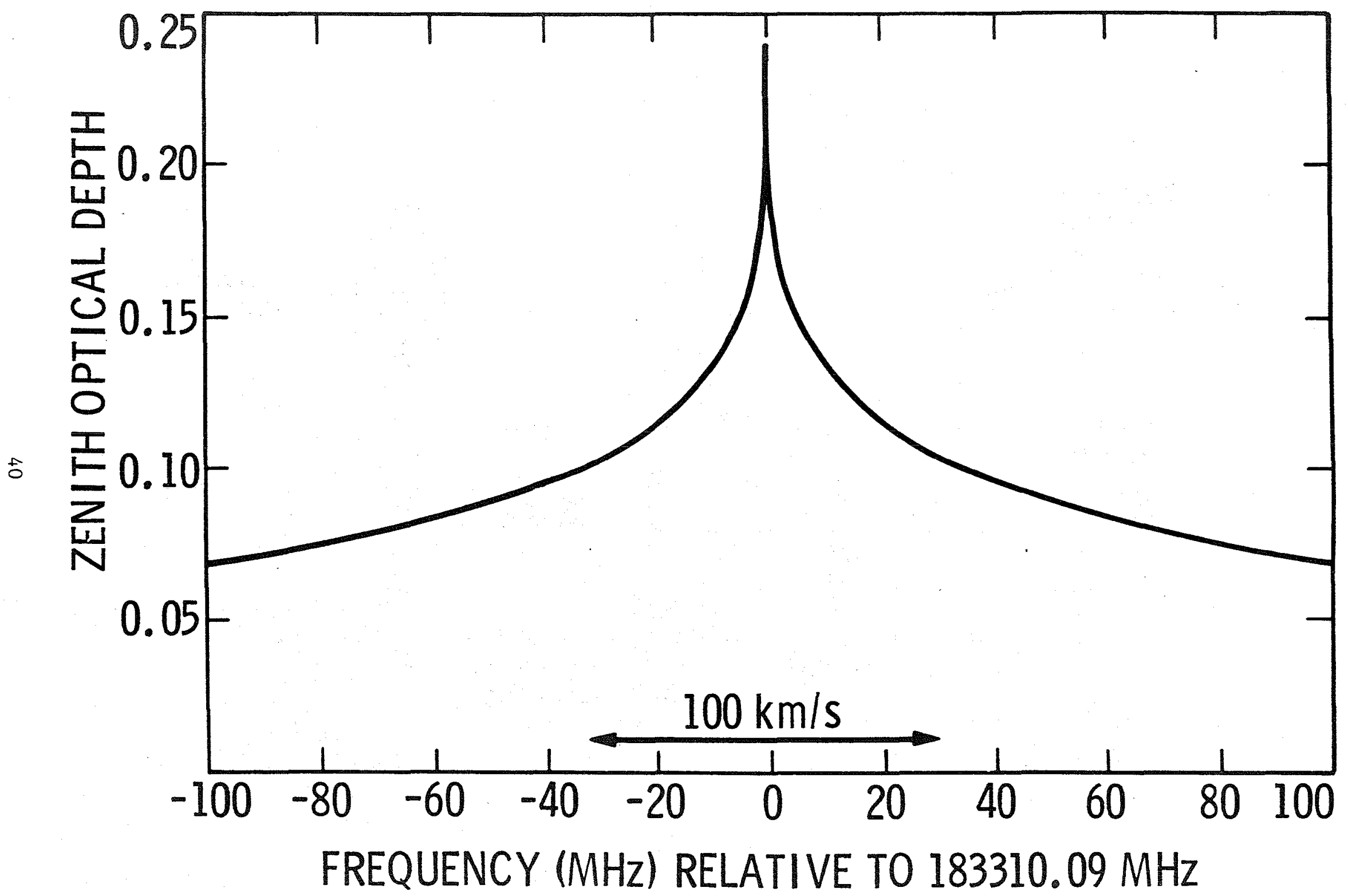

Fig. 2 


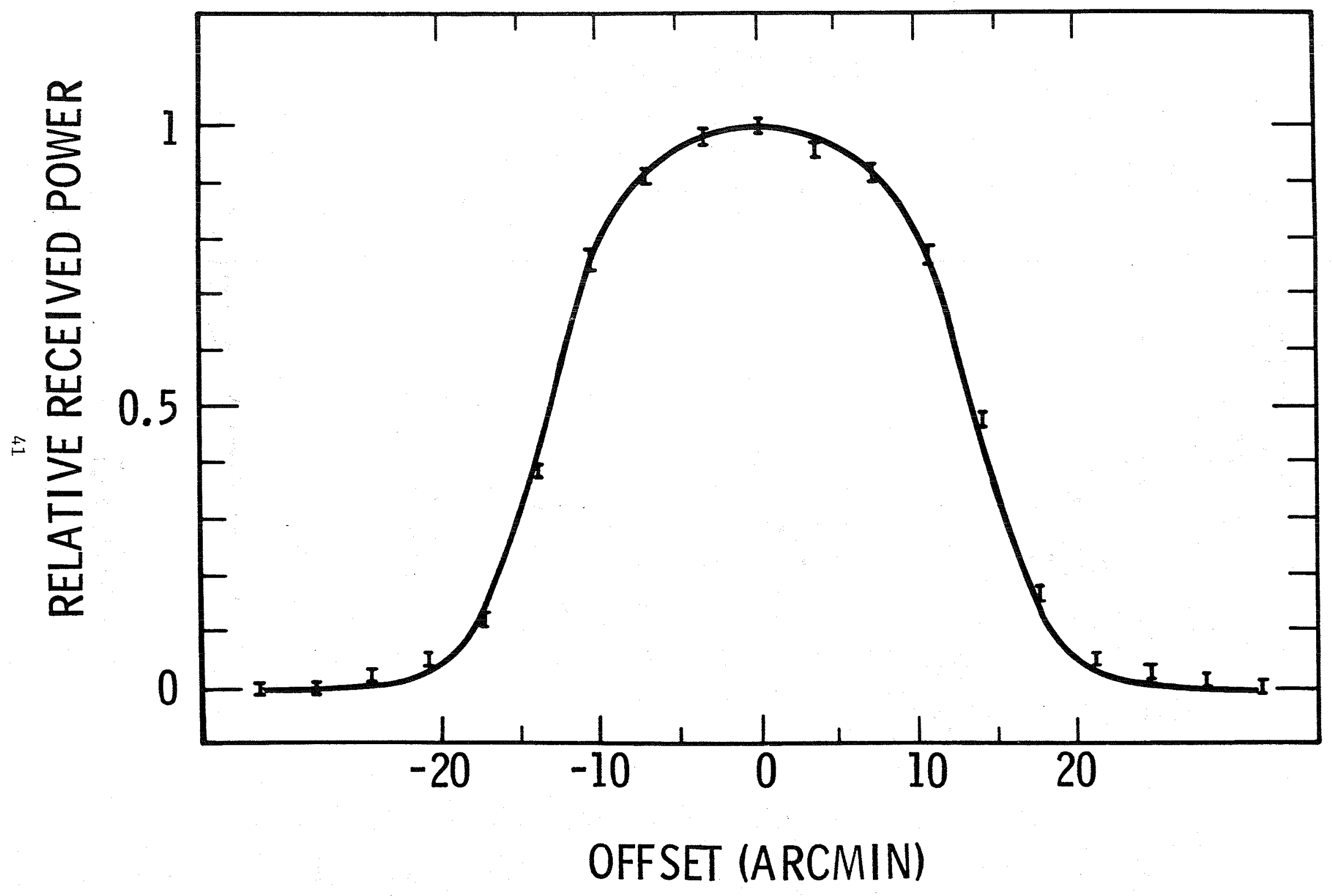

Fig. 3 


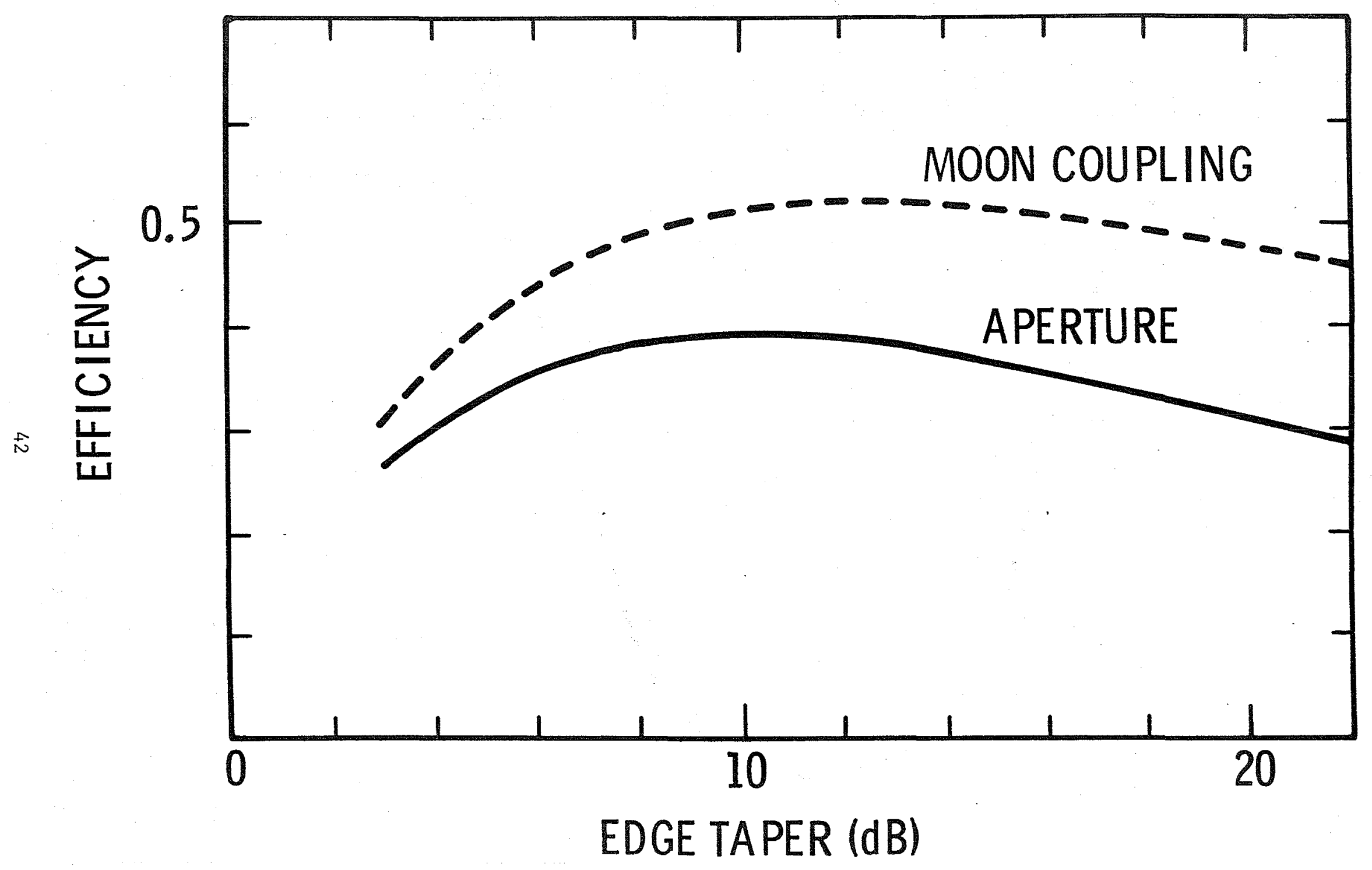

Fig. 4 


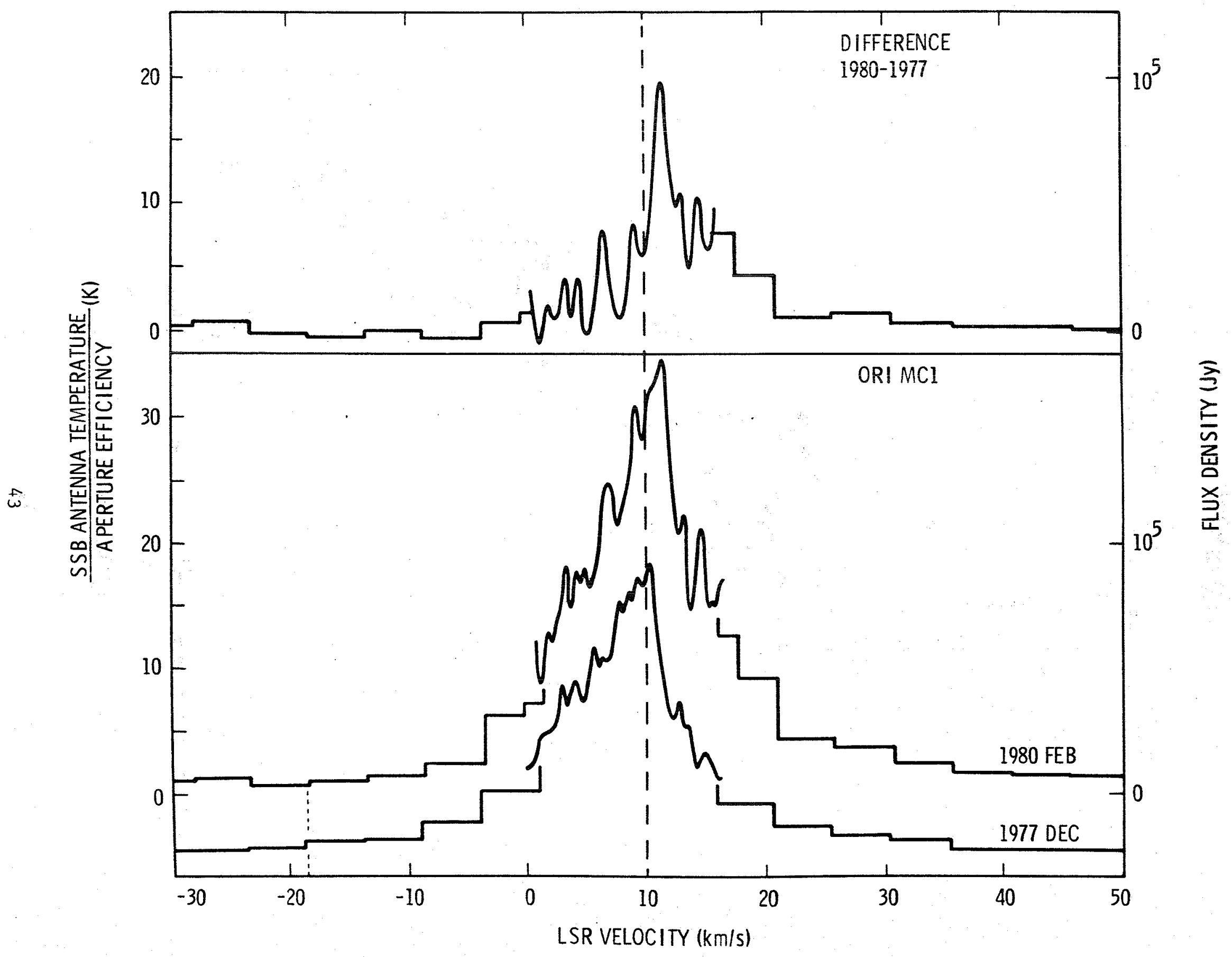

Fig. 5 


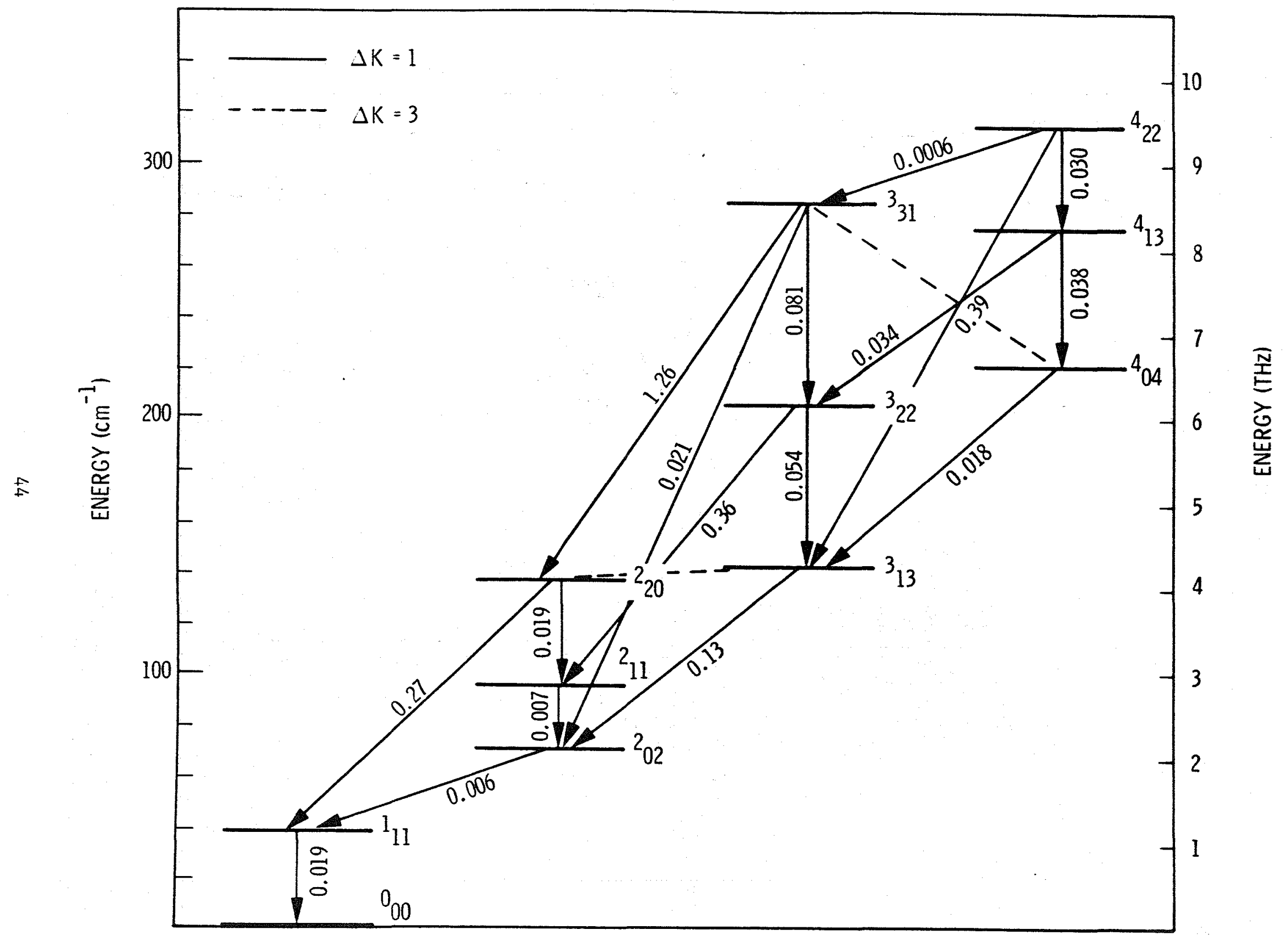

Fig. 6 


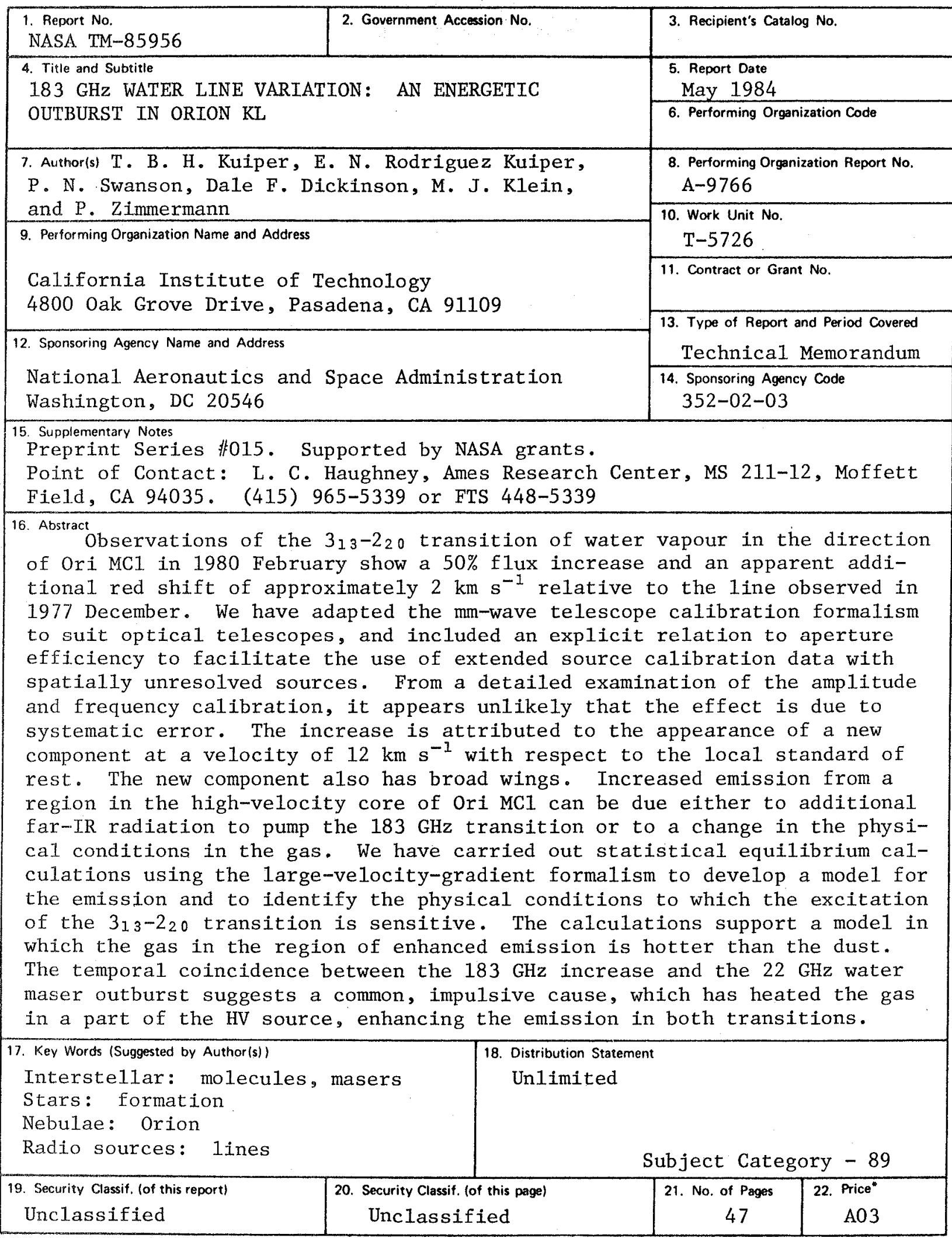

"For sale by the National Technical Information Service, Springfield, Virginia 22161 
End of Document 